\section{Nützen nachhaltige Geldanlagen?}

\author{
Mit der Rentenreform wurde eine ethische, soziale und ökologische Berichts- \\ pflicht für Altersvorsorgeprodukte neu eingeführt. Dies wird dem Markt für nach- \\ haltige Geldanlagen einen weiteren kräftigen Impuls verleihen. Damit wird die \\ Frage immer interessanter, was nachhaltige Geldanlagen tatsächlich bewirken.
}

$\mathrm{D}$ Von Walter Kablenborn agen in Deutschland beläuft sich inzwischen auf etwa 5 Milliarden Euro. Die Wachstumsraten des Marktes liegen im oberen zweistelligen Bereich, und jeden Monat kommt ein neuer Öko-Fonds hinzu. Neben spezialisierten Anbietern entdecken auch immer mehr große Finanzdienstleister diese Produktsparte - und dies wird mit der Rentenreform noch zunehmen.

Damit wird nun die Frage immer interessanter, was nachhaltige Geldanlagen tatsächlich für die Umwelt bewirken. In einem Gutachten haben Bundesumweltministerium und Umweltbundesamt diese Frage weltweit erstmalig systematisch untersuchen lassen (1).

Am meisten beachtet werden normalerweise die Finanzierungseffekte zugunsten umwelffreundlicher Unternehmen. So können nachhaltige Geldanlagen eine Anschubfinanzierung erbringen und zu einer Verbesserung der allgemeinen Finanzierungsbedingungen beitragen. Der Effekt der Anschubfinanzierung betrifft vor allem den Bereich der alternativen Energieerzeugung. Hier sind inzwischen erhebliche Erfolge zu verzeichnen; praktisch der gesamte Windkraftsektor in Deutschland verdankt seine Existenz den ökologischen Geldanlagen. Aber auch in anderen Sektoren, wie etwa dem Verlagswesen, sind mit ökologischen Geldanlagen wirtschaftlich rentable und für den Umweltschutz bedeutsame Initiativen ermöglicht worden.

Eine Verbesserung der Finanzierungsbedingungen kann sich zum Beispiel aus einer Steigerung des Aktienkurses von umwelffreundlichen Unternehmen oder über zinsvergünstigte Kredite ergeben, die über ökologische Geldanlagen ermöglicht werden. Beides spielt in der Praxis aber kaum eine Rolle. Das Volumen der zinsvergünstigten Kredite liegt nur im unteren zweistelligen Millionenbereich und Auswirkungen von ökologischen Geldanlagen auf die Aktienkurse sind lediglich bei einigen kleineren Unternehmen am Markt überhaupt möglich.
So wichtig Finanzierungseffekte daher insgesamt sein mögen, der Umweltnutzen, der sich hier ergibt, ist begrenzt. Insbesondere ist die mitunter geäußerte Kritik richtig, dass der Erwerb von Umweltaktien oder Öko-Fonds zumeist kaum positive Finanzierungsauswirkungen für umwelffreundliche Unternehmen hat. Die daraus gezogene Schlussfolgerung, Umweltaktien oder Öko-Fonds hätten kaum positive Effekte für die Umwelt (2), ist aber falsch.

Dies ergab eine Befragung sämtlicher in Deutschland vertriebener Öko-Fonds sowie einer erheblichen Anzahl von Unternehmen im Portfolio dieser Fonds. Für Umweltaktien und insbesondere für Öko-Fonds lassen sich unter anderem folgende indirekte Effekte belegen:

- Verbesserungen bei Umweltdatensammlungen und der Umweltberichterstattung in Unternehmen,

- die Stärkung von Umweltabteilungen (Personalausstattung und Durchsetzungskraft),

- die Einführung von Umweltmanagementsystemen und Umweltprogrammen, sowie

- die Definition interner Umweltziele und Modifikationen beim internen Benchmarking.

Als bedeutsam wurde von vielen der befragten Unternehmen ferner hervorgehoben, dass die Tatsache, als nachhaltige Geldanlage gehandelt zu werden, sich als positiv für ihr Image erweist.

Insgesamt lassen sich drei wesentliche Schlussfolgerungen ziehen:

1. Nachhaltige Geldanlagen haben deutliche positive Umweltauswirkungen, die in ihrem Ausmaß nicht mehr als unerheblich eingestuft werden können, auch wenn sie diffus und nicht quantifizierbar sind. Die Wirkungsmechanismen sind dabei ausgesprochen vielfältig und komplex und eine Reduktion allein auf die Finanzierungswirkung wird den wahren Sachverhalten nicht gerecht.

2. Der Umweltnutzen einer Geldanlage korrespondiert nicht mit einem Renditeverzicht. Auch lukrative ökologische Geldanlagen können durchaus einen deutlichen Beitrag zum Umweltschutz leisten.

\section{- Nicht nur Finanzierungseffekte}

3. Nicht der "ökologische Reinheitsgrad“ einer Geldanlage entscheidet über ihren Umweltnutzen. Ein Beleg dafiur, dass ausgerechnet immer die ,saubersten" ökologischen Geldanlagen, also etwa die Öko-Fonds, die die strengsten Auswahlkritierien anlegen, am meisten für die Umwelt ausrichten, hat sich durch die Untersuchung nicht ergeben. Im Gegenteil, bei einigen durchaus auch öffentlich als nicht ganz ,ökologisch“ kritisierten Anlagen konnten interessante Nutzeffekte festgestellt werden. Vor diesem Hintergrund erscheint die gegenwärtige Praxis, Öko-Fonds im Wesentlichen an dem Inhalt ihrer Portfolios zu bemessen (3), fragwürdig. Diese Erkenntnis ist speziell auch für die zunehmend aufkommende Debatte um ein Labeling von nachhaltigen Geldanlagen von Bedeutung.

Kritisch ist abschließend anzumerken, dass Anbieter und Vermittler nachhaltiger Geldanlagen, aber auch Käufer sich in der Vergangenheit noch zu wenig um die Umweltauswirkungen gekümmert haben. Die Transparenz am Markt ist diesbezïglich relativ gering. Eine Steigerung des Umweltnutzens von ökologischen Geldanlagen ist in vielen Fällen noch möglich.

Ganz besonders betrifft dies die Frage der direkten Beeinflussung des Umweltmanagements. Weder auf Hauptversammlungen (sharebolder activism) noch im direkten Diskurs (engagement) versuchen Investoren in ökologische Geldanlagen bisher, ihre Rechte und Möglichkeiten als Aktionäre voll auszunutzen und auf Unternehmen einzuwirken. Während dies im Ausland inzwischen in großem Maße erfolgt und dabei auch klare Erfolge zu verzeichnen sind, ist dieses Vorgehen in Deutschland fast unbekannt.

\section{Anmerkungen}

(1) Das Gutachten ist zusammen mit anderen Untersuchungen zum Thema nachhaltige Geldanlagen als UBA-Texteband 36/01 erschienen.

(2) Vgl. etwa Pfeiffer, Hermannus: Grüne Geldanlagen. Geld anlegen mit ökologischer und sozialer Verantwortung. Köln 1995; Deml, Max u.a.: Rendite ohne Reue. Frankfurt am Main 1996.

(3) Vgl. Brian, Marcus: Umwelffonds. In: Ökonomy, ÖkoTest Ausgabe Mai 2000.

\section{Der Autor}

Walter Kahlenborn ist als Berater für verschiedene nationale und internationale Organisationen tätig. Kontakt: Nassauische Str. 26, 10717 Berlin.

Tel. 030/86420-833, Fax -834,

E-mail: office@kahlenborn.de 
(c) 20I0 Authors; licensee IÖW and oekom verlag. This is an article distributed under the terms of the Creative Commons Attribution Non-Commercial No Derivates License (http://creativecommons.org/licenses/by-nc-nd/3.o/), which permits unrestricted use, distribution, and reproduction in any medium, provided the original work is properly cited. 